\title{
Prescription Rates of Cardiovascular Medications in a Large UK Primary Care Chronic Kidney Disease Cohort
}

Rupert Major ${ }^{1,2}$, David Shepherd², Graham Warwick ${ }^{1}$, Nigel Brunskill ${ }^{1,3}$

${ }^{1}$ Department of Nephrology, Leicester General Hospital, Leicester UK ${ }^{2}$ Department of Health Sciences, University of Leicester, Leicester, UK ${ }^{3}$ Department of Infection, Immunity and Inflammation, University of Leicester, Leicester, UK

Short Title: Prescription Rates of Cardiovascular Medications in Chronic Kidney Disease

Word Count: 2,843

Number of References: 27

Correspondence to: Dr Rupert Major

National Institute for Health Research Academic Clinical Fellow,

Department of Nephrology,

Leicester General Hospital,

Gwendolen Road,

Leicester,

LE5 4PW

UK

Email: $\quad$ rm157@le.ac.uk

Tel:

+441162588043

Fax:

+441162584764 


\section{Abstract}

Background and Aims: Chronic kidney disease (CKD) is associated with increased cardiovascular risk. Guidelines have suggested the universal use of statins in CKD but aspirin's role is less well defined. The aim of this study was to determine prescription rates for statins and aspirin in a UK CKD cohort and to establish factors that influenced prescription rates.

Methods: We used data from a UK primary care CKD cohort to study rates of prescription of statins and aspirin. Simple rates were initially calculated. Binary logistic regression was utilised with either statin or aspirin prescription as the outcome variable and co-variates including demographic details and co-morbidities.

Results: There were 31,056 individuals in the cohort with at least one estimated glomerular filtration rate (eGFR) of $<60 \mathrm{ml} / \mathrm{min} / 1.73 \mathrm{~m}^{2} .65 .1 \%$ individuals had 2 eGFR results $<60 \mathrm{ml} / \mathrm{min} / 1.73 \mathrm{~m}^{2}$ more than 3 months apart. Mean eGFR at baseline was $51.1 \mathrm{ml} / \mathrm{min} / 1.73 \mathrm{~m}^{2}$ (SD $9.1 \mathrm{ml} / \mathrm{min} / 1.73 \mathrm{~m}^{2}$ ). $64.9 \%$ had a diagnosis of hypertension, $18.8 \%$ had diabetes mellitus and $29.8 \%$ a history of cardiovascular disease. Statins were prescribed to 14,972 (48.2\%) and aspirin to $11,023(35.5 \%)$. The regression model suggested that cardiovascular disease, hypertension and diabetes mellitus all influenced prescriptions of statins and aspirin but overall CKD stage, calculated by either eGFR or proteinuria, did not.

Conclusions: Prescriptions of statins and aspirin in CKD is based more on the presence of co-morbidities than the CKD severity. Further physician and patient education of the increased cardiovascular risk associated with chronic kidney disease and its suitability for cardiovascular medication intervention is required. 


\section{Background}

Chronic kidney disease (CKD) affects up to $8 \%$ of the UK population [1], but only within the last decade has it been convincingly recognised as a condition associated with higher cardiovascular risk compared to the general population [2]. Most recently, CKD has been recognised as a coronary heart disease equivalent for future cardiovascular (CV) events, with risk in excess to that of diabetes mellitus (DM) [3].

However, specific evidence for treatment of cardiovascular disease within CKD, particularly in relation to primary prevention, is limited. Concerns exist in the context of reduced renal function regarding safety and excessive side effects of commonly used CV medications, such as statins and aspirin. Within CKD, statins have been hypothesised to increase the risk of acute kidney injury [4], and aspirin the risk of bleeding events [5]. Further, lipid profile abnormalities become increasingly disparate as CKD advances in severity [6] and surrogate measurements such as low density lipoprotein reduction may be more difficult to interpret in CKD. Most clinical trials exclude patients with 'severe' CKD [7], commonly defined as an estimated glomerular filtration rate (eGFR) or creatinine clearance in of $<$ of $<60 \mathrm{ml} / \mathrm{min} / 1.73 \mathrm{~m}^{2}$. This limits the applicability of the evidence to many CKD patients. Furthermore, nephrology may be the most under-represented medical subspeciality in clinical trials [7]. Consequently, the ability of primary and secondary care physicians to make evidence based decisions regarding primary and secondary CV risk prevention in CKD patients is restricted. Recently, National Institute for Health and Care Excellence (NICE) [8] and Kidney Disease Improving Global Outcomes (KDIGO) [9] guidelines have recommended the use of statins for all individuals with CKD. However, there remains limited evidence for the use of aspirin, particularly for primary prevention in CKD.

Given these uncertainties it is possible that opportunities to manage CV risk factors in primary care may be passed over. Using a large database of CKD patients identified in primary care, this study aimed to establish the prescription rates of the commonly used CV medications, statins and aspirin.. The use of these medications alongside common CV related co-morbidities was also assessed. 


\section{Methods}

We analysed the baseline cross-sectional data from 'The Primary-Secondary Care Partnership to Prevent Adverse Outcomes in Chronic Kidney Disease' (PSP-CKD) study (ClinicalTrials.gov Identifier: NCT01688141). PSP-CKD is a cluster randomised controlled trial of CKD management in primary care. The study is approved by the local Research Ethics Committee. The intervention is a nurse practitioner-led CKD management programme in primary care with secondary care nephrology support versus usual primary care management, essentially general practitioner (GP) led management of CKD. Randomisation is at the level of the general practice and individual patient consent was not sought. In total 49 practices were recruited from Northamptonshire, UK and completed participation in the trial. The trial commenced in 2010 with the extraction of baseline data before practices were randomly allocated to either the intervention or control groups. This paper reports the study's baseline data.

A web based CKD management and audit software tool, IMproving Patient care and Awareness of Kidney disease progression Together (IMPAKT) [10], was developed to identify all CKD patients in participating practices from practice electronic medical records. For eGFR data, IMPAKT used Morbidity Information Query and Export Syntax (MIQUEST) search methodology to analyse the practice record of all adult patients retrospectively back to 2005 for any eGFR $<60 \mathrm{ml} / \mathrm{min} / 1.73 \mathrm{~m}^{2}$ and, where more than one eGFR value $<60 \mathrm{ml} / \mathrm{min} / 1.73 \mathrm{~m}^{2}$ was available, calculated the time interval between results. Individuals either receiving maintenance dialysis or with a renal transplant were excluded. For other biomedical data related to these patients IMPAKT extracted data most temporally close to the practice randomisation date. For medications, IMPAKT extracted the relevant information if it had been prescribed within six months of the extraction date. This paper reports the baseline data for the PSP CKD study prior to any interventions.

For the purposes of the study an anonymised dataset from each practice was exported to University of Leicester Clinical Trials Unit to assemble a prospective CKD database from all participating practices. The data comprises anthropometric, demographic, relevant medical history, prescribed medications, blood and urine test 
results.

eGFRs were reported using the MDRD equation [11]. Proteinuria data was derived from two sources, urine dipstick results and urine protein quantification by either albumin creatinine ratio (ACR) or protein creatinine ratio (PCR). Where both ACR and PCR were available the latter was used for classification due to its better calibration to the gold standard, 24 hour urine protein measurement [12]. Individuals were assigned to a CKD stage based on KDIGO guidelines for both eGFR and proteinuria [13]. CV disease includes any individual with a Read code diagnosis of previous ischaemic heart disease, stable angina, cerebrovascular accident, transient ischaemic attack or heart failure. Read codes are nationally standardised medical codes used in UK primary care (see supplement).

Data are reported for continuous outcomes as mean \pm standard deviation (SD) and for categorical variables as counts and percentage. Statin and aspirin prescriptions were the outcomes of interest. Simple unadjusted prescription rates were calculated. Binary logistic regression was performed using the prescription of either statins or aspirin as the outcome variables. The regression models were calculated across the whole cohort and also for individuals without any pre-existing cardiovascular disease, the primary prevention cohort. Gender, age, CKD stage based on eGFR and proteinuria quantification and co-morbidities were used as co-variables. Confirmed CKD, the 'CKD cohort', refers to individuals who had at least two eGFRs $<60 \mathrm{ml} / \mathrm{min} / 1.73 \mathrm{~m}^{2}$ measured at least 3 months apart [13].

Initially eGFR and age were considered as categorical variables, CKD stage and age group by decade, and then as continuous variables, eGFR in $\mathrm{ml} / \mathrm{min} / 1.73 \mathrm{~m}^{2}$ and age in years. Other variables remained unchanged. For proteinuria stage, CKD stage and age group categorical co-variates, stage $A 1$, stage $3 A$ and $<50$ years respectively were used as the reference groups. All statistical analysis was performed using Statistical Package for the Social Sciences (SPSS) 22.0 (IBM), with $p<0.05$ taken to reflect statistical significance. 


\section{Results}

The records of 353,256 registered patients $\geq 18$ years of age from 49 practices were analysed. The baseline cohort data of the PSP-CKD study included 31,056 (8.8\%) individuals with at least 1 eGFR $<60 \mathrm{ml} / \mathrm{min} / 1.73 \mathrm{~m}^{2}$, referred to henceforth as the 'eGFR cohort'. Of the eGFR cohort, $65.1 \%$ of patients $(20,276$ individuals) had 2 or more eGFR results $<60 \mathrm{ml} / \mathrm{min} / 1.73 \mathrm{~m}^{2}>3$ months apart and are henceforth referred to as the 'CKD cohort'. The CKD cohort have 'true' CKD as defined by KDIGO and represent $5.7 \%$ of the total practice population $\geq 18$ years of age. In the eGFR cohort 9,291 (29.9\%) individuals had a coded history of CV disease. Table 1 shows the full baseline descriptors of the eGFR cohort.

The eGFR cohort had a mean eGFR at baseline of $51.1 \mathrm{ml} / \mathrm{min} / 1.73 \mathrm{~m}^{2}$ (SD 9.1 $\mathrm{ml} / \mathrm{min} / 1.73 \mathrm{~m}^{2}$ ) and $80.9 \%$ had CKD stage $3 \mathrm{~A}$. Table 2 shows the frequency and percentage of overall CKD stage based on eGFR and proteinuria, including information on missing proteinuria data, in the eGFR cohort. 20,169 (64.9\%) had a coded diagnosis of hypertension and 5,819 (18.8\%) a coded diagnosis of DM. Mean systolic blood pressure was $133.8 \mathrm{mmHg}(\mathrm{SD} 16.3 \mathrm{mmHg})$ and mean diastolic blood pressure $75.4 \mathrm{mmHg}$ (SD $10.0 \mathrm{mmHg}$ ). Altogether 3,230 (10.4\%) of the eGFR cohort were current smokers and 11,353 (36.6\%) ex-smokers.

In the eGFR cohort, 14,972 (48.2\%) were prescribed statins and 11,023 (35.5\%) individuals aspirin. $73.2 \%$ of individuals with known CV disease were prescribed a statin compared to $37.6 \%$ without known CV disease (Pearson $X^{2}<0.001$, OR 4.53, $95 \% \mathrm{Cl} 4.30$ to 4.78). A similar relationship occurred for aspirin (Pearson $X^{2}<0.001$, OR $7.46,95 \% \mathrm{Cl} 7.07$ to 7.88 ). Higher rates of prescriptions for both medication groups were found for patients with HTN, DM or with $\geq 2$ recorded eGFRs (Figure 1) $<60 \mathrm{ml} / \mathrm{min} / 1.73 \mathrm{~m}^{2}\left(X^{2}<0.001\right.$ for all) compared to those without the condition. Statin prescription rates varied significantly across age groups, peaking in the 70-79 years group (58.7\%), whilst decreasing as age both increased and decreased. Figure 2 shows statin and aspirin prescription rates by age groups. Rates of statin prescription increased in the eGFR cohort as CKD stage worsened. There was a statistically significant trend when CKD stage was used as a categorical variable $(p<0.01$ for linear-by-linear association). When eGFR was used as a continuous 
variable a similar correlation was found ( $\beta$ co-efficient $-0.016, p<0.001)$. Mean total cholesterol was lower in the statin group $(4.5 \mathrm{mmol} / \mathrm{L}$, mean difference $0.88 \mathrm{mmol} / \mathrm{L}$, $\mathrm{p}<0.001,95 \% \mathrm{Cl} 0.85-0.91 \mathrm{mmol} / \mathrm{L})$.

Binary logistic regression was performed for statin and aspirin prescription (Table 3). For binomial co-variates, all tested variables (CV disease, HTN, DM, confirmed CKD and male gender) were all associated with statins and aspirin prescriptions ( $p$-value $\leq 0.001$ for all). Age groupings in the regression model showed a similar pattern to percentage of prescription in the initial comparison. The peak group was again the 70-79 age bracket (OR $3.20, p<0.001$ for both medication groups), with a similar decrease in the odds ratio as the ages increased and decreased. When eGFR was treated as a continuous variable, a $1 \mathrm{ml} / \mathrm{min} / 1.73 \mathrm{~m}^{2}$ change in eGFR was not significantly associated with statin prescription $(\beta$ co-efficient $-0.003, p=0.09$ ) but was associated with aspirin prescription ( $\beta$ co-efficient $-0.011, p<0.001$ ). 


\section{Discussion}

The PSP-CKD study of patients with eGFRs $<60 \mathrm{ml} / \mathrm{min} / 1.73 \mathrm{~m}^{2}$ was derived from a large number of general practices in Northamptonshire, and is likely to be broadly typical of UK primary care CKD patients.

The results of the current study suggest that both statins and aspirin are widely used in CKD. Furthermore the use of both medications is significantly increased in association with DM, HTN and established CV disease in CKD patients. All these comorbidities are positively associated with future CV events, with CV disease and CKD the most influential [3]. However, the regression analysis suggests that severity of CKD has, at best, a minor influence on rates of prescriptions for these medications even though CV risk increases as CKD progresses [13]. Only stage 5 CKD showed significantly higher rates of prescriptions in the regression models. This may be linked to these patients being more likely to be managed in secondary care.

Overall the prescription rates for both statins and aspirin in this cohort of CKD patients, particularly in those with DM or pre-existing CV disease, are lower than would be expected if relevant guidelines were fully implemented $[8,9,13,14,15]$. This finding is in agreement with previous studies describing disappointing levels of primary care prescribing in DM and for primary and secondary prevention of CV disease $[16,17,18,19,20]$. In England the Quality and Outcomes Framework rewards primary care for the appropriate use of statins and aspirin in relevant conditions, and providing such financial incentives has been shown to be effective in the management of DM [21]. The current analysis indicates room for substantial improvement in prescribing for management of CV risk in CKD. Established CVD, DM and HTN are all well established factors that influence aspirin and statin prescriptions for the purpose of reducing CVD events [16,20]. The low prescription rates for statins and aspirin in uncomplicated CKD, compared to CKD associated with DM, CV disease and/or hypertension in the current study suggests that CKD is not widely regarded as a key risk factor for CV disease in its own right. This is further highlighted by the absent association of worsening CKD with statin and aspirin prescriptions. 
The use of statins to lower CV morbidity and mortality in CKD is well established $[22,23,24,25]$. The role of aspirin in CKD is less clear. Evidence for its use in CKD is limited and appears to show limited benefit with possible increased risk of bleeding [26]. Observational data also suggests that aspirin may increase cardiovascular risk and bleeding risk [27]. Therefore whilst higher levels of CV disease may make universal use of aspirin in CKD an attractive proposition an increased risk of bleeding may negate any benefit.

The PSP-CKD cohort identified using IMPAKT has similar characteristics to that previously described in the NEOERICA study [1]. Both studies utilise similar methodology to identify CKD and associated co-morbidities from primary care electronic records. Co-morbidities are similar in both cohorts with hypertension being present in approximately two thirds of individuals and DM in around $20 \%$ in PSPCKD and $14 \%$ in NEOERICA. Both cohorts consisted of approximately $60 \%$ females and had a similar mean age in the low seventies.

However, unlike NEOERICA, the PSP-CKD study was able to identify individuals where the diagnosis of CKD was confirmed by at least 2 eGFRs $<60 \mathrm{ml} / \mathrm{min} / 1.73 \mathrm{~m}^{2}$ more than 3 months apart. In PSP-CKD all adults with a single eGFR $<60$ $\mathrm{ml} / \mathrm{min} / 1.73 \mathrm{~m}^{2}$ were identified, and of these nearly two thirds had at least one additional eGFR of $<60 \mathrm{ml} / \mathrm{min} / 1.73 \mathrm{~m}^{2}$ in an appropriate time period. The use of a single eGFR to diagnose and classify CKD will tend to overestimate prevalence. Differences in methodology therefore explain the reported true CKD prevalence of $5.7 \%$ in PSP-CKD compared to $8.5 \%$ in NEOERICA. Interestingly, based on a single eGFR $<60 \mathrm{ml} / \mathrm{min} / 1.73 \mathrm{~m}^{2}$, the prevalence of CKD in PSP-CKD is $8.9 \%$ and very similar to NEOERICA. The current data indicate that around $30 \%$ of primary care patients with a single low eGFR require repeat testing. One of the key drivers underlying the various CKD clinical guidelines is to mitigate the $\mathrm{CV}$ risk associated with CKD. Therefore it is of considerable importance to assess whether opportunities to intervene with risk modification strategies are fully exploited.

The use of IMPAKT has facilitated the accumulation of a rich database of primary care CKD patients. However this analysis has a number of limitations. The 
participating general practices, although large in number, were not randomly selected and thus may not be fully representative of the UK population. Further, the primary purpose of this work was not to study medication prescription rates and therefore it is subject to similar limitations and risk of bias common to post-hoc analyses. Also, observational data may not account for other confounding factors that may have influenced the prescription rates of these medications. Prescription data within electronic records does not equate to medication dispensing or adherence by the patient. The dataset also had no internal audit function to verify the accuracy of coding of medical conditions within the electronic records. This is most likely to underestimate the prevalence of comorbidities if conditions have not been correctly coded in individual records. Approximately a third of our cohort did not have a second, and confirmatory, eGFR $<60 \mathrm{ml} / \mathrm{min} / 1.73 \mathrm{~m}^{2}$. Whilst initial univariate analysis suggested that not confirming the diagnosis of CKD with a follow-up eGFR might influence the decision to prescribe either medications, this effect was reduced in the multivariate analysis. This suggests that individuals with co-morbidities such as DM or pre-existing CVD were more likely to have confirmatory eGFR checked.

Overall, the current data suggests that statin and aspirin use in CKD is based more on the presence of co-morbidities, and particularly the presence of $\mathrm{CV}$ disease, than CKD severity, based on either eGFR or proteinuria. Further education of the increased cardiovascular risk associated with CKD and its suitability for cardiovascular medication intervention may increase prescription rates and improve CV outcomes in CKD. 
Funding: This research was funded by the National Institute for Health Research (NIHR) Collaboration for Leadership in Applied Health Research and Care (CLAHRC) East Midlands. Dr Major is currently a Kidney Research UK Clinical Fellow. The views expressed are those of the authors and not necessarily those of Kidney Research UK, the NHS, the NIHR or the Department of Health. 


\section{References}

1. Stevens $P, O^{\prime}$ Donoghue $D$, de Lusignan S, Van Vlymen J, Klebe B, Middleton $\mathrm{R}$ et al. Chronic kidney disease management in the United Kingdom: NEOERICA project results. Kidney Int. 2007;72(1):92-99.

2. Go A, Chertow G, Fan D, McCulloch C, Hsu C. Chronic Kidney Disease and the Risks of Death, Cardiovascular Events, and Hospitalization. New England Journal of Medicine. 2004;351(13):1296-1305.

3. Tonelli M, Muntner P, Lloyd A, Manns B, Klarenbach S, Pannu N et al. Risk of coronary events in people with chronic kidney disease compared with those with diabetes: a population-level cohort study. The Lancet. 2012;380(9844):807-814.

4. Dormuth C, Hemmelgarn B, Paterson J, James M, Teare G, Raymond C et al. Use of high potency statins and rates of admission for acute kidney injury: multicenter, retrospective observational analysis of administrative databases. BMJ. 2013;346(mar18 3):f880-f880.

5. Stroke and Bleeding in Atrial Fibrillation with Chronic Kidney Disease. New England Journal of Medicine. 2012;367(23):2262-2262.

6. Shlipak M, Fried L, Cushman M. Cardiovascular Mortality Risk in Chronic Kidney Disease: Comparison of Traditional and Novel Risk Factors. ACC Current Journal Review. 2005;14(7):14-15.

7. Coca S, Krumholz H, Garg A, Parikh C. Underrepresentation of Renal Disease in Randomized Controlled Trials of Cardiovascular Disease. JAMA. 2006;296(11):1377.

8. National Institute for Health and Care Excellence. Lipid modification: cardiovascular risk assessment and the modification of blood lipids for the primary and secondary prevention of cardiovascular disease. 2014.

9. Wanner C, Tonelli M, Cass A, Garg A, Holdaas H, Jardine A et al. KDIGO Clinical Practice Guideline for Lipid Management in CKD: summary of recommendation statements and clinical approach to the patient. Kidney Int. 2014;85(6):1303-1309.

10.IMproving Patient care and Awareness of Kidney disease progression Together (IMPAKT), Available at: http://www.impakt.org.uk/ [Accessed 14th July 2015]. 
11. Levey A. A More Accurate Method To Estimate Glomerular Filtration Rate from Serum Creatinine: A New Prediction Equation. Annals of Internal Medicine. 1999;130(6):461.

12. Chitalia VC, Kothari J, Wells EJ, Livesey JH, Robson RA, Searle M, Lynn KL Cost-benefit analysis and prediction of 24-hour proteinuria from the spot urine protein-creatinine ratio. Clin Nephrol. 2001;55(6):436.

13. Stevens P. Evaluation and Management of Chronic Kidney Disease: Synopsis of the Kidney Disease: Improving Global Outcomes 2012 Clinical Practice Guideline. Annals of Internal Medicine. 2013;158(11):825.

14. National Institute for Health and Care Excellence. Type 2 diabetes: The management of type 2 diabetes. 2009.

15. National Institute for Health and Care Excellence. Cardiovascular risk assessment and lipid modification. 2015.

16. Wu J, Zhu S, Yao G, Mohammed M, Marshall T. Patient Factors Influencing the Prescribing of Lipid Lowering Drugs for Primary Prevention of Cardiovascular Disease in UK General Practice: A National Retrospective Cohort Study. PLoS ONE. 2013;8(7):e67611.

17.Bennett KE, Williams D, Feely J. Under-prescribing of cardiovascular therapies for diabetes in primary care. Eur J Pharmacol 58: 835-841, 2003

18. Simmons R, Carlsen A, Griffin S, Charles M, Christiansen J, Borch-Johnsen K et al. Variation in prescribing of lipid-lowering medication in primary care is associated with incidence of cardiovascular disease and all-cause mortality in people with screen-detected diabetes: findings from the ADDITION-Denmark trial. Diabet Med. 2014;31(12):1577-1585.

19. Mainous A, Tanner R, Shorr R, Limacher M. Use of Aspirin for Primary and Secondary Cardiovascular Disease Prevention in the United States, 20112012. Journal of the American Heart Association. 2014;3(4):e000989e000989.

20.Wei L. Statin use in the secondary prevention of coronary heart disease in primary care: cohort study and comparison of inclusion and outcome with patients in randomised trials. BMJ. 2005;330(7495):821-0.

21. Gallagher N, Cardwell C, Hughes C, O'Reilly D. Increase in the pharmacological management of Type 2 diabetes with pay-for-performance in primary care in the UK. Diabet Med. 2014;32(1):62-68. 
22. Baigent C, Landray M, Reith C, Emberson J, Wheeler D, Tomson C et al. The effects of lowering LDL cholesterol with simvastatin plus ezetimibe in patients with chronic kidney disease (Study of Heart and Renal Protection): a randomised placebo-controlled trial. The Lancet. 2011;377(9784):2181-2192.

23.Palmer S, Craig J, Navaneethan S, Tonelli M, Pellegrini F, Strippoli G. Benefits and Harms of Statin Therapy for Persons With Chronic Kidney Disease. Annals of Internal Medicine. 2012;157(4):263.

24. Upadhyay A, Earley A, Lamont J, Haynes S, Wanner C, Balk E. LipidLowering Therapy in Persons With Chronic Kidney Disease. Annals of Internal Medicine. 2012;157(4):251.

25. Major R, Cheung C, Gray L, Brunskill N. Statins and Cardiovascular Primary Prevention in CKD: A Meta-Analysis. Clinical Journal of the American Society of Nephrology. 2015;10(5):732-739.

26. Saito Y, Morimoto T, Ogawa H, Nakayama M, Uemura S, Doi N et al. LowDose Aspirin Therapy in Patients With Type 2 Diabetes and Reduced Glomerular Filtration Rate: Subanalysis from the JPAD trial. Diabetes Care. 2011;34(2):280-285.

27.Kim A, Lim H, Ro H, Ko K, Han S, Chang J et al. Low-Dose Aspirin for Prevention of Cardiovascular Disease in Patients with Chronic Kidney Disease. PLoS ONE. 2014;9(8):e104179. 


\section{Figure Legend}

1. Rates of statin and aspirin prescriptions by presence of co-morbidities in eGFR cohort.

2. Rates of statin and aspirin prescriptions by age groups.

\section{Table Legend}

1. Descriptors of the whole PSP-CKD Study eGFR cohort, including those with and without known cardiovascular disease. Abbreviations - CV cardiovascular, CKD - chronic kidney disease, eGFR - estimated glomerular filtration rate, units $\mathrm{ml} / \mathrm{min} / 1.73 \mathrm{~m}^{2}$. '\%' refers to the percentage of either the whole eGFR cohort, or those with and without CV disease. Results are expressed as mean $\pm \mathrm{SD}$, or by per cent as appropriate.

2. Frequencies and percentage (in brackets) of whole PSP-CKD Study eGFR cohort by GFR and proteinuria stage.

3. Odds Ratios and $p$ values with $95 \%$ confidence intervals in brackets of mulitvariate logistic regression model for eGFR Cohort. Outcome variable - statin or aspirin prescription. All co-variates and reference groups for the model are shown. $\mathrm{Cl}$ - confidence interval. 
Table 1

\begin{tabular}{|c|c|c|c|}
\hline Category & No CV Disease & CV Disease & All Individuals \\
\hline Number of patients & 21765 & 9291 & 31056 \\
\hline Female & $14394(66)$ & $4838(52)$ & $19232(62)$ \\
\hline Age, years & $71.0 \pm 13.1$ & $78.3 \pm 9.6$ & $73.2 \pm 12.6$ \\
\hline \multicolumn{4}{|l|}{ Smoking Status } \\
\hline Never & $11993(55)$ & $4249(46)$ & $16242(52)$ \\
\hline Ex-smoker & $7235(33)$ & $4118(44)$ & $11353(37)$ \\
\hline Current smoker & $2389(11)$ & $841(9)$ & $3230(10)$ \\
\hline No data & $148(1)$ & $83(1)$ & $231(1)$ \\
\hline \multicolumn{4}{|l|}{ CKD Stage by MDRD eGFR } \\
\hline G3A & $18277(84)$ & $6850(74)$ & $25127(81)$ \\
\hline G3B & $2476(11)$ & 1779 (19) & $4255(14)$ \\
\hline G4 & $514(2)$ & $460(5)$ & $974(3)$ \\
\hline G5 & $172(1)$ & $70(1)$ & $242(1)$ \\
\hline No data & $326(2)$ & $132(1)$ & $458(2)$ \\
\hline MDRD eGFR, $\mathrm{ml} / \mathrm{min} / 1.73 \mathrm{~m}^{2}$ & $51.9 \pm 8.6$ & $49.2 \pm 10.0$ & $51.1 \pm 9.1$ \\
\hline \multicolumn{4}{|l|}{ CKD Stage by Proteinuria } \\
\hline $\mathrm{A} 1$ & $11776(54)$ & $4901(53)$ & $16677(54)$ \\
\hline $\mathrm{A} 2$ & $1601(7)$ & $995(11)$ & $2596(8)$ \\
\hline $\mathrm{A} 3$ & $1622(8)$ & $897(10)$ & $2519(8)$ \\
\hline No data & $6766(31)$ & $2498(27)$ & $9264(30)$ \\
\hline Protein Creatinine Ratio & $33.8 \pm 99.4$ & $38.9 \pm 102.9$ & $35.6 \pm 100.7$ \\
\hline Albumin Creatinine Ratio & $7.7 \pm 35.7$ & $8.9 \pm 23.9$ & $8.2 \pm 31.8$ \\
\hline \multicolumn{4}{|l|}{ Co-morbidities } \\
\hline HTN & $13704(63)$ & $6465(70)$ & $20169(65)$ \\
\hline DM & $3545(16)$ & $2274(25)$ & 5819 (19) \\
\hline Systolic Blood Pressure, mmHg & $134.7 \pm 16.0$ & $131.8 \pm 17.0$ & $133.8 \pm 16.3$ \\
\hline Diastolic Blood Pressure, $\mathrm{mmHg}$ & $76.6 \pm 9.7$ & $72.7 \pm 10.2$ & $75.4 \pm 10.0$ \\
\hline \multicolumn{4}{|l|}{ Medication Prescriptions } \\
\hline Statin & $8174(38)$ & $6798(73)$ & $14972(48)$ \\
\hline Aspirin & $4747(22)$ & $6276(68)$ & $11023(35)$ \\
\hline
\end{tabular}




\section{Table 2}

\begin{tabular}{llllll}
\hline & \multicolumn{5}{c}{ Proteinuria Stage } \\
& \multicolumn{1}{c}{ A1 } & \multicolumn{1}{c}{ A2 } & A3 & No Data & Total \\
\hline \multicolumn{1}{c}{ eGFR Stage } & & & & & \\
3A & $14029(45)$ & $1832(6)$ & $1717(6)$ & $7549(24)$ & $25127(81)$ \\
$3 B$ & $2115(7)$ & $570(2)$ & $469(2)$ & $1101(4)$ & $4255(14)$ \\
4 & $352(1)$ & $148(1)$ & $220(1)$ & $254(1)$ & $974(3)$ \\
5 & $47(<1)$ & $31(<1)$ & $77(<1)$ & $87(<1)$ & $242(1)$ \\
No eGFR Data & $134(<1)$ & $15(<1)$ & $36(<1)$ & $273(1)$ & $458(2)$ \\
\hline Total & $16677(54)$ & $2596(8)$ & $2519(8)$ & $9264(30)$ & 31056
\end{tabular}


Table 3

\begin{tabular}{|c|c|c|c|c|}
\hline & \multicolumn{2}{|l|}{ Statin } & \multicolumn{2}{|l|}{ Aspirin } \\
\hline & OR (95\% Cl) & $p$ value & OR $(95 \% \mathrm{Cl})$ & $\mathrm{p}$ value \\
\hline Known CV Disease & $4.64(4.37-4.93)$ & $<0.01$ & $6.04(5.70-6.39)$ & $<0.01$ \\
\hline HTN & $2.01(1.90-2.12)$ & $<0.01$ & $1.48(1.40-1.57)$ & $<0.01$ \\
\hline DM & $3.21(2.99-3.44)$ & $<0.01$ & $1.56(1.46-1.67)$ & $<0.01$ \\
\hline$\geq 2$ eGFRs $<60$ & $1.44(1.36-1.53)$ & $<0.01$ & $1.29(1.21-1.37)$ & $<0.01$ \\
\hline Male & $1.20(1.14-.1 .26)$ & $<0.01$ & $1.27(1.20-1.34)$ & $<0.01$ \\
\hline \multicolumn{5}{|c|}{ Age Group (<50 years reference) } \\
\hline $50-59$ & $1.94(1.65-2.30)$ & $<0.01$ & $1.45(1.18-1.80)$ & $<0.01$ \\
\hline $60-69$ & $2.93(2.52-3.42)$ & $<0.01$ & $2.45(2.02-2.97)$ & $<0.01$ \\
\hline $70-79$ & $3.20(2.75-3.73)$ & $<0.01$ & $3.20(2.65-3.86)$ & $<0.01$ \\
\hline $80-89$ & $1.89(1.62-2.20)$ & $<0.01$ & $3.29(2.72-3.97)$ & $<0.01$ \\
\hline $90+$ & $0.72(0.60-0.86)$ & $<0.01$ & $3.30(2.68-4.06)$ & $<0.01$ \\
\hline \multicolumn{5}{|c|}{ Proteinuria Stage (A1 reference) } \\
\hline A2 & $0.97(0.88-1.06)$ & 0.48 & $1.02(0.93-1.13)$ & 0.64 \\
\hline A3 & $0.96(0.88-1.06)$ & 0.46 & $1.03(0.93-1.13)$ & 0.62 \\
\hline \multicolumn{5}{|c|}{ eGFR Stage (3A reference) } \\
\hline 3B & $0.97(0.90-1.05)$ & 0.47 & $1.10(1.02-1.19)$ & 0.01 \\
\hline 4 & $1.16(1.00-1.35)$ & 0.05 & $1.37(1.18-1.59)$ & $<0.01$ \\
\hline 5 & $1.70(1.27-2.29)$ & $<0.01$ & $2.48(1.85-3.33)$ & $<0.01$ \\
\hline
\end{tabular}




\section{List of Abbreviations}

CKD - chronic kidney disease

CV - cardiovascular

DM - diabetes mellitus

eGFR - estimated glomerular filtration rate

GP - general practitioner

IMPAKT - IMproving Patient care and Awareness of Kidney disease progression

Together

IBM - International Business Machines

KDIGO - Kidney Disease Improving Global Outcomes

MIQUEST - Morbidity Information Query and Export Syntax

NEOERICA - NEw Opportunities for Early Renal Intervention by Computerised

Assessment

NICE - National Institute for Health and Care Excellence

PSP-CKD - The Primary-Secondary Care Partnership to Prevent Adverse Outcomes

in Chronic Kidney Disease

SD - standard deviation

SPSS - Statistical Package for the Social Sciences

UK - United Kingdom 
Figure 1

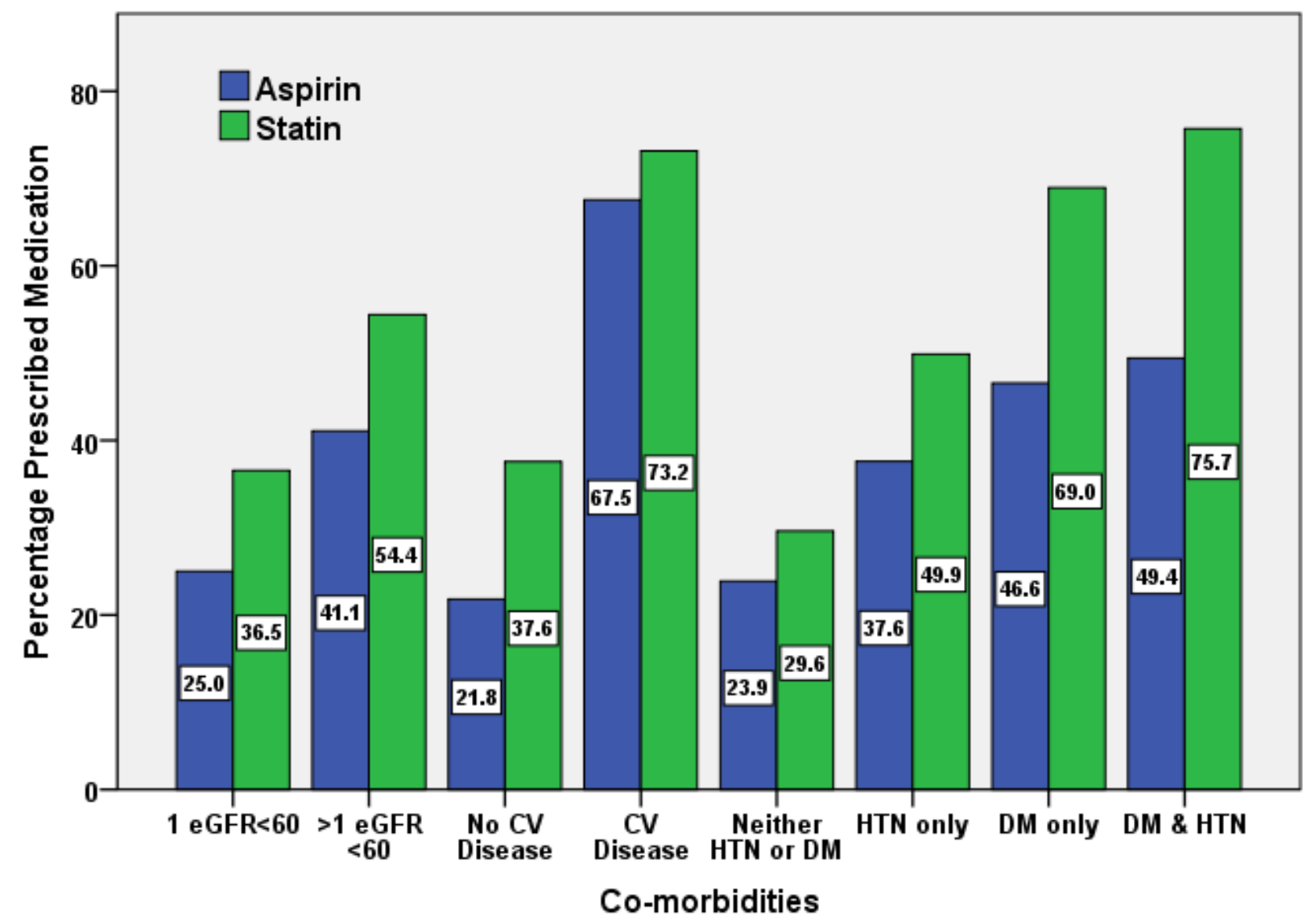


Figure 2

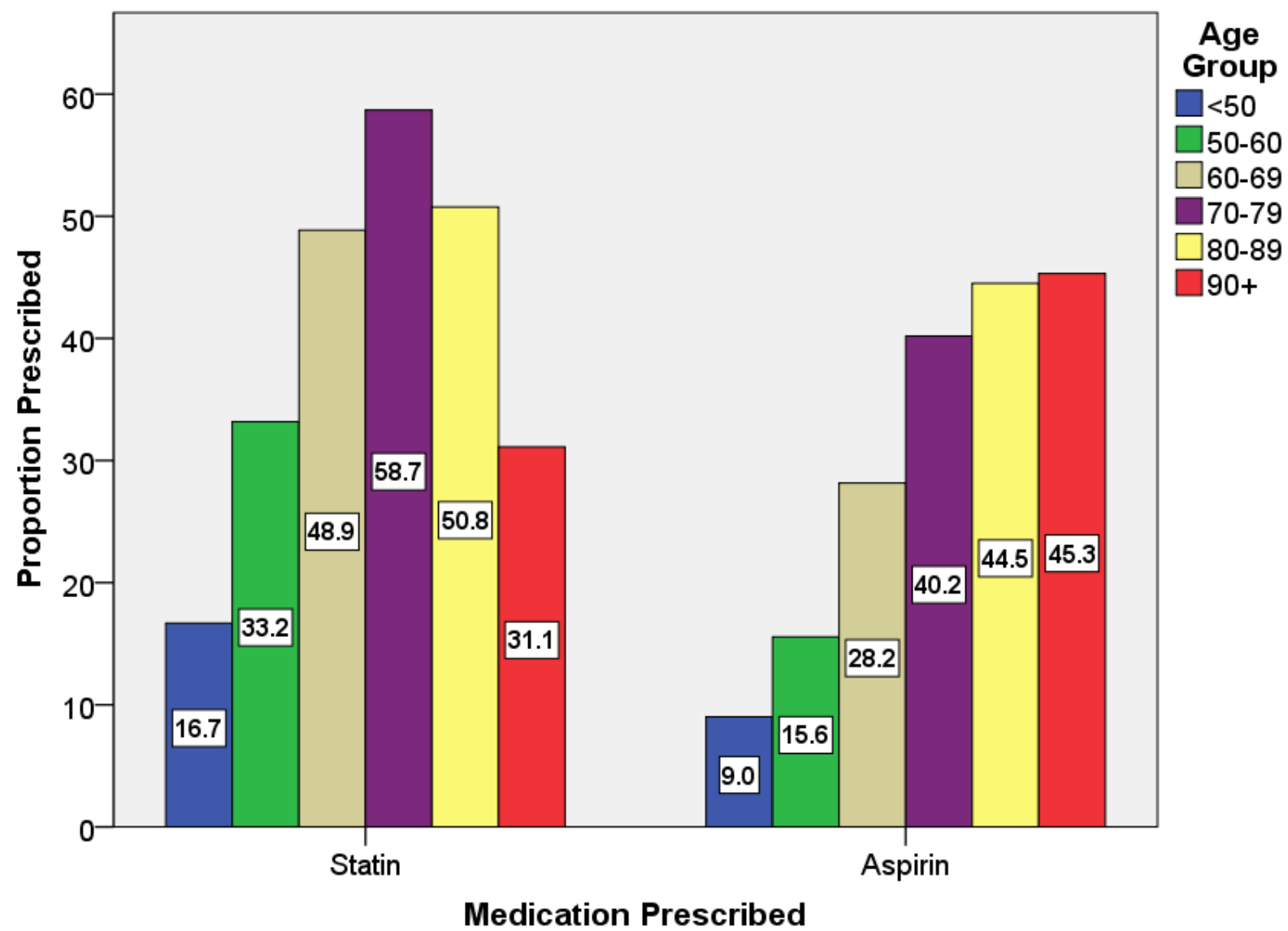

